PSICOLOGIA Vol. VIII NN 21990

\title{
PSICOTERAPIA RACIONAL EMOTIVA
}

Víctor Amoros*

En el presente artículo el autor realiza una síntesis didáctica sobre los origenes, fundamentos, características y la técnica de la terapia racional emotiva de Albert Ellis. Se explica cómo las personas crean sus propios trastomos emocionales y conductuales por sus prejuicios y pensamientos equivocados. Se concluye afirmando que son las ideas irracionales las que originan en gran medida las neurosis. El trabajo se detiene en la actitud del terapeuta quien aplica una técnica directiva reforzando los componentes cognitivos del paciente.
The following paper briefly describes the origin, basis, characteristics and technic of the emotional rational therapy of Albert Ellis. The author describes how persons create their own emotional and behavioural disturbs based on their wrong prejudgment and thoughts. He concludes asserting that irrational ideas in a special way origin neurosis. This work emphasizes the therapist's actitude that applies a directive technic reforcing the patient's cognitive components. 

El ser humano tiene un sistema de conocimientos y creencias que conforman su área cognitiva y se combina con sus emociones y reacciones conducturales. Para explicamos nuestra manera de actuar, es necesario que nos detengamos èn los pensamientos que vamos estructurando en el transcurso de la vida y que están estrechamente vinculados a nuestros sentimientos y actitudes. En consecuencia la manera de pensar es realmente importante en la expresión de las emociones y el comportamiento normal y perturbado. Pero si bien es cierto que nuestros pensamientos y creencias afectan de manera significativa nuestras emociones y conductas, estas últimas dejan también su huella en nuestro pensamiento. Es decir, se da una constante interrelación.

La terapia racional emotiva (RET) afirma que las personas no reaccionan directamente en forma emocional o conductual ante los acontecimientos que encuentran en su vida, sino son ellas mismas las que causan sus reacciones según la forma en que interpretan o valoran los acontecimientos que experimentan. Y lo que es más importante, nuestras creencias irracionales básicas son las causantes de nuestras perturbaciones emocionales. Igualmente la evaluación que hacemos de nosotros mismos es importante, porque si no tomamos en cuenta nuestras necesidades y preferencias y tratamos de reemplazarlas por las demandas y obligaciones, nos enfermamos. Si nos valoramos en forma negativa y tenemos una autoimagen pobre de nosotros mismos, afectamos nuestra personalidad.

Para la RET la psicoterapia es un procedimiento que conduce a que el paciente se explique racionalmente su realidad interna y aquella que la rodea y a que logre un mayor desarrollo e integración de su personalidad. En el transcurso de esta terapia se utilizan los procedimientos logicos empíricos conducentes a preguntar, debatir y poner en duda las ideas irracionales; a que los pacientes reconozcan lo absurdo de sus creencias y luego adopten otras más reales y apropiadas. Este enfoque psicológico no se detiene ni en la historia pasada del paciente ni en sus actuales trastornos, sino en las ideas irreales y en las interpretaciones exageradas que el paciente hace de sus experiencias. Por consiguiente cuando nos emocionamos y actuamos, siempre tenemos algunos pensamientos relacionados con nuestros sentimientos y actitudes. 
Albert Ellis creó la RET en 1955 y la consideró como una teoría de la personalidad y de la práctica psicoterapéutica y la sustentó en el esquema del A-B-C, donde una experiencia activadora (A) no causa un comportamiento (C), sino lo que hay que cambiar es (B); el sistema individual de ideas y creencias.

El aporte de Ellis es haber enfatizado lo cognitivo y conductual creando una teoría independiente de otros autores que ya utilizaban una terapia directiva y orientada hacia lo racional (Mahoney 1988). Asimismo es una terapia que sirve para tratar principalmente trastornos emocionales.

\section{Origen}

Los antecedentes de la RET los encontramos en los escritos de Adler y en algunos otros terapeutas alejados un tanto de Freud tales como: Karen Horney, Franz Alexander, Thomas French, Eric Berne y Harry Stack Sullivan. Ellis fue psicoanalista, posteriormente se desilusiono de la libre asociación, interpretación de los sueños, los mecanismos de resistencia y la transferencia; así como de la actitud pasiva del terapeuta mientras el paciente luchaba con sus síntomas. En relación a la transferencia solía afirmar que no a todos los pacientes les preocupa el terapeuta, sino esencialmente quieren resolver lo mejor y más rápido posible sus problemas. Igualmente reparó - lo que es fácil comprobar en la práctica profesional- que no es suficiente que la persona tome conciencia de sus dificultades para que éstas se solucionen, porque sus temores y conflictos persisten. Al respecto escuchemos a Ellis: ....muchos de mis pacientes seguían agarrándose a sus temores y hostilidades. ¿Por qué seres humanos tan inteligentes, incluidos los que han seguido una introspección psicologica, se agarran de forma tan desesperada a sus ideas irracionales sobre si mismos y sobre los demás? ¿Por qué se siguen culpando de esa manera tan ilogica y exagerada (dando origen así a la ansiedad, culpabilidad y depresión) y culpando implacablemente a los demás?".

Otra observación importante que hizo Ellis fue reparar que el ser humano, a diferencia de los animales, no sólo es frustrado o recompensado por sus sensaciones, sino también es sancionado o gratificado por sus procesos simbólicos: es decir por su propio pensamiento; con una connotación importante: esta forma de pensar está desligada de las gratificaciones o sanciones del mundo exterior. Así por ejemplo: los perros temen a los estímulos nocivos reales, mientras que los seres humanos tememos a los estímulos desagradables, tanto imaginados o pensados como reales.

Asimismo creemos lo que se dice de nosotros mismos, consideramos horroroso que se nos desapruebe y llegamos a creer con facilidad lo que piensan de nosotros. En relación a los animales éstos son condicionados a temer los efectos de los castigos físicos y a través de sus temores físicos aprenden a tener 
miedo a gestos y palabras. En el caso de los seres humanos, además de temer a los castigos físicos, así como a los gestos y palabras, también nos desalientan sólo estos últimos aún cuando no vayan acompañados de castigo físico; con lo cual "llegamos a tener miedo de nuestros procesos puramente verbales o de otro tipo de señalización". No sólo aprendemos de los demás y de los medios de comunicación de la sociedad a temer las palabras, pensamientos y gestos de nuestros semejantes, sino nuestras propias señalizaciones o conversaciones. Debido a esta capacidad de temer los gestos y la comunicación verbal de los demás y la nuestra, podemos imaginar o vivenciar temores que no tienen ninguna base en castigos físicos o sensoriales.

Muchos de los temores que observamos en los pacientes neuróticos son ansiedades transmitidas que más tarde las hicieron suyas. Así por ejemplo nos dice Ellis, primero se le dice a una persona que esta sancionada o que no es amado y que esto es terrible; en seguida la misma persona se dice a sí misma que ser rechazado es espantoso. La repetición de este hecho constituye el origen de muchas perturbaciones emocionales. Pero sobre todo los autoadoctrinamientos verbales, porque el hombre no es sólo un animal sugestionable, sino autosugestionable; en consecuencia en forma enérgica seguimos readoctrinándonos. En general los pacientes no sólo están adoctrinados con ideas irracionales y falsas, sino por inercia y en forma automática siguen cogidos a estas ideas negativas originales hasta incorporarlas a su filosofía de la vida. Este asirse a la irracionalidad se produce dé manera inconsciente, pero también en forma voluntaria. Así seguimos creyendo en muchas ideas irracionales o simples definiciones irracionales. Durante la práctica terapéutica se comprueba una resistencia del paciente a dejar de ser neurotico. Ellis nos recuerda que así como el lenguaje nos permite ser seres humanos - hablar con otros y con nosotros mismos - nos lleva también a abusar de esta posibilidad definiendo cosas como terribles, cuando en el peor de los casos, sólo son inconvenientes o molestas. Asimismo reparó que más importante que desaparecer lo hecho, era anular lo dicho o pensando. Y se detuvo en las exclamaciones que los pacientes se decían a si mismo creando sus perturbaciones y conflictos : ¡Es terrible haberme equivocado! ¡Es horroroso que me desaprueben! ¡Qué pueden pensar de mí!.

El hecho de vivenciar emociones negativas intensas no causadas por el dolor físico — como el miedo y la hostilidad - son el resultado de la ignorancia o de la perturbación; en consecuencia deben eliminarse aplicando el conocimiento o la razón; una persona no siempre se siente triste o alegre, por objetos o acontecimientos externos, sino que pueden ser sus percepciones, ideas y actitudes interiorizadas la fuente de su perturbación.

Esta realidad ya fue expuesta en la antiguedad por los filósofos estoicos; en especial por Zenón, Crisipo, Panateo de Rodas (quien introdujo el estoicismo 
en Roma); igualmente por Cicerón, Séneca, Epicteto y Marco Aurelio. Fue Epicteto quien en el siglo I después de Cristo afirmó: los hombres no se preocupan por las cosas, sino por las opiniones que de ellas tienen. Y Shakespeare lo repite en Hamlet cuando afirma: no existe nada que sea bueno o malo, es el pensamiento el que lo hace así.

\section{Características}

La RET insiste, aún sin certeza absoluta, en los factores orgánicos que se dan en la perturbación del comportamiento humano. Afirma que la alteración emocional proviene de profundas y complejas tendencias biológicas como de influencias sociales; que el aprendizaje de la primera infancia interviene en el desorden psicológico, pero tiene un efecto más inmediato en la personas innatamente más vulnerables; por último que el condicionamiento social y la labor terapéutica ayudan muchísimo a las personas perturbadas, incluso cuando poseen dificultades causadas por una predisposición innata. Esta terapia postula que los pensamientos y deseos inconscientes pueden ser afectados por métodos lógicos y didácticos; se presenta de esta manera como un proceso reeducativo cuyo objeto es ayudar a que una persona corrija sus conceptos errados, y que abandone sus viejos hábitos de pensar irracionalmente adquiriendo nuevos modelos de pensar en forma racional. Esto requiere un duro aprendizaje práctico y constante con la exigencia que aprendamos a elevar nuestro nivel de tolerancia a la frustración.

Ellis sostiene que las personas se crean sus propios trastornos cognitivos, emocionales y conductuales; que las neurosis se originan y fortalecen en gran parte por las ideas irracionales. Inclusive las personas muy inteligentes pueden tender por su herencia biologica, a ser muy sugestionables, a generalizar y pensar irrazonablemente. La RET se presenta como una método psicológico basado en la capacidad que tenemos de pensar racionalmente, de anticiparnos a los hechos con un mayor auto control, disminuir la angustia y alcanzar nuestras metas, motiva la auto realización y se esfuerza por demostrar a las personas sus erróneas exigencias absolutas porque el trastorno mental y emocional del ser humano se producen en gran parte por exagerar la importancia $o$ seriedad de las cosas. En consecuencia el terapeuta realiza un esfuerzo directo y didáctico por desaparecer las ideas erradas y ayuda a que los pacientes las sustituyan por puntos de vista más sanos y eficaces, minimizando los sentimientos de vergüenza y de auto culpabilidad.

Respecto a la actitud del terapeuta, ésta es directiva; cree que sólo un ataque directo y permanente sobre las ideas irracionales del paciente sostenidas durante mucho tiempo, podrá arrancarlas de raíz; igualmente es optimista en su esfuerzo por cambiar el comportamiento humano; le va demostrando al paciente como sus creencias e ideas sin base real son las responsables de su 
angustia y conflictos en sus relaciones interpersonales y no por efecto de alguna experiencia infantil traumática a nivel inconsciente.

\section{Fundamento de la RET}

Para la terapia racional emotiva es el pensamiento el que da características de bueno o malo a los actos humanos; asimismo nos neurotizamos porque estamos emocionalmente incapacitados para pensar con mayor claridad. Todo cambio verdadero de la personalidad sólo puede llevarse a cabo, si la persona aprende a reconocer sus percepciones y pensamientos irreales y se esfuerza por transformarlos por una filosofía de vida más lógica y razonable. Sin este cambio en sus presupuestos filosóficos e ideológicos, ninguna transformación profunda de su personalidad tendrá lugar. Afirma que las creencias, actitudes y objetivos de vida vienen a ser formas de pensamiento; en consecuencia sólo una persona con ideas claras es capaz de descubrir sus mecanismos de auto-engaño y sólo los procesos racionales pueden descubrir las áreas de la irracionalidad. Asimismo cree que estamos continuamente readoctrinándonos con filosofías de vida negativas; es necesario entonces examinar, comprender, desafiar y cuestionar estas filosofías negativas.

La RET afirma que poseemos cuatro procesos básicos: percepción, movimiento, pensamiento y emoción; todos están íntimamente interrelacionados. La emoción y el pensamiento no son dos procesos dispares, sino tienen coincidencias significativas; igual los procesos sensitivo y motor, tienen una interrelación constante. En consecuencia, las cuatro funciones: sensitiva, motora, emotiva y cognitiva o pensante, no se experimentan de manera aislada. Por ejemplo dice Ellis: veo una pelota, quiero atraparla o jugar con ella; se producen sentimientos: me agrada o disgusta; pienso sobre ella: me recuerda a una pelota que tuve o me imagino lo que puedo hacer con esta pelota. Es decir, cuando actuó me emocionó y pienso y comprometo a los demás procesos de conducta de manera consciente o inconsciente.

La emoción se controla con el pensamiento gran parte de lo que llamamos emoción, no es más que un tipo de pensamiento que puede contener muchos prejuicios; como se ha expresado, es muy difícil separar las emociones y el pensamiento; así como la motivación del aprendizaje. Las emociones tienen un componente cognoscitivo y una expectativa o disposición a actuar. Para la RET el ser humano está condicionado desde los primeros años de su vida a tener miedo de algo, preferentemente de sus padres; ahora bien, para la terapia psicoanalítica es necesario ver qué ocurrió en el origen de su vida; en cambio la terapia racional emotiva repara en que ha sido enseñado a tener miedo; pero ahora ya no es un niño y no necesita tener miedo a la misma cosa (reprobación de sus padres). Se espera que el temor condicionado o neurosis desaparezca. 
La introspección durante los primeros condicionamientos puede invalidar de alguna forma los efectos de este proceso. Ellis creía que estaba cerca de una aproximación entre el psicoanálisis freudiano y la teoría del aprendizaje conductista: "Empecé a ver que no era probable que la introspección sola logrará que el individuo superara sus temores y hostilidades tan profundamente arraigados. Necesitaba una etapa de acción combatiendo el temor y la hostilidad". Asimismo si se obliga a las personas a hacer lo que temen, pronto comprenderán que eso no es tan temible como erróneamente pensaban que era: su temor llegará a ser descondicionado o suprimido. Este pensamiento no es original de Ellis, si recordamos que ya Adler lo había puesto en práctica con algunos de sus pacientes (Adler, 1959). Igualmente Viktor Frankl (1984) máximo exponente de la psicoterapia humanista con su técnica de la intención paradojica.

Como terapeuta, Ellis no sólo enseñaba a sus pacientes el origen de sus miedos, sino que hicieran lo que temían como exponerse al rechazo de sus padres o de otras personas para que experimentaran en forma concreta que estas vivencias ya no eran temibles en la actualidad; dice: "En lugar de psicoterapeuta de orientación psicoanalítica, empecé a ser mucho más ecléctico, exhortativo, persuasivo y activo-directivo. Y encontré que este tipo de terapia, aun con sus limitaciones concretas, tenía mucho más éxito con la mayoría de mis pacientes que mis métodos psicoanalíticos anteriores".

Los síntomas que uno produce como el insomnio, cefaleas, etc. no siempre se generan por los estímulos o activadores exteriores, sino por lo que nos decimos a nosotros mismos (B) ante dichos estímulos; en consecuencia lo que crea el desequilibrio psíquico para esta terapia, es nuestro sistema de creencias en relación a los estímulos o elementos activadores. En otras palabras no nos perturban las cosas o los sucesos, sino la opinión que tenemos de ellos. (C) no siempre sigue a (A); lo que hay que buscar y en todo caso cambiar es (B): el sistema individual de ideas y creencias. El esquema de la RET es el siguiente:

A: acontecimiento activador, no siempre es una causa directa.

C: consecuencia emocional y conductal.

B: nuestras creencias acerca de A.

Otro aspecto fundamental de este enfoque terapéutico consiste en afirmar que la valoración que las personas hacen de sí mismas, generalmente es irreal; se creen buenas o malas totalmente. Es necesario valorar con objetividad nuestras cualidades, aptitudes y defectos; pero también disfrutar la existencia más que justificarla. Esta filosofía nos conduce a revisar nuestros "deberes patologicos", discutirlos y cambiarlos por pensamientos como "sería mejor o sería preferible". Desde este punto de vista son las personas las que generalmente se crean sus propios transtornos emocionales porque mantienen firmemente sus creencias y filosofías erróneas muy arraigadas en ellas mismas. 
El terapeuta racional no asigna importancia al historial pasado del paciente ni lo relaciona con sus trastornos actuales; no cree que el paciente se puso neurótico por sus experiencias anteriores, sino por su pocos realistas y superexigentes nterpretaciones de estas experiencias. Emplea escaso tiempo en conocer o interpretar el pasado de la persona a quien ayuda psicologicamente, ensenándole modelos prácticos en su vida diaria. Por ejemplo, es preferible tratar a los demás en forma considerada; yo puedo generalizar esta creencia y convertirla en irracional y dogmática (Bs): porque es preferible para mí tratar a los demás de manera considerada, debo hacerlo todo el tiempo al margen que sea una persona indigna.

El terapeuta RET reconoce que la herencia biologica del paciente y su condicionamiente social facilitan ciertos hábitos anormales de conducta y formas de auto-sabotaje. Pero acrecienta la fe del paciente para que pueda cambiar con la práctica necesaria para vivir con un mínimo de ansiedad y hostilidad; se esfuerza a fin de que el paciente acepte que cuando algo desagradable o frustrante no se puede evitar, lo razonable es aceptarlo en lugar de continuar refiriéndose a sus aspectos negativos. Igualmente se postula que el cariño a sí mismo y el hacer lo que en verdad se quiere en la vida es mucho más importante que estar atento a lo que hagan los demás. Si no se lleva a cabo un cambio fundamental en los presupuestos filosóficos e ideológicos, ningún cambio profundo de personalidad tendrá lugar; al transformarse el pensamiento, se produce el cambio de las emociones.

La RET da énfasis a la lógica, la razón y la objetividad. Acepta a los seres humanos como falibles y limitados y afirma que no es suficiente la introspección para encontrar el origen de los síntomas neuroticos, porque sólo con la introspección no se superan estos síntomas. Lo importante es conseguir el cambio en las actitudes con uno mismo y los demás. El terapeuta demuestra a los pacientes que sus autoverbalizaciones han sido y continúan siendo el origen de sus perturbaciones emocionales, convenciéndolo de la verdad de frases más racionales y menos autodestructivas, recordándole que todos podemos vivenciar pensamientos y comportamientos irracionales.

Ciertamente existen condiciones ambientales negativas que a veces producen comportamientos irracionales en personas normalmente sanas. Para la RET este tipo de reacción sẹ produce porque se tiene alguna disposición básica para expresar actitudes irracionales cuando experimentan tensión. Pero, en general todas las personas a menudo tienden a actuar en forma irracional por que tenemos una tendencia tanto biologica como cultural. Para Ellis hay una inclinación humana básica a tener dos creencias contradictorias al mismo tiempo: una intelectual que se mantiene débil y otra emocional vigorosa y consistente. Se actúa de acuerdo a esta última. La tendencia a tener creencias contradictorias simultáneas parece que es parte de la condición humana. 
De acuerdo a la RET tenemos muchas creencias, cogniciones, pensamientos o ideas (Bs) en relación a los sucesos activadores. Las (Bs) se orientan a extender su influencia poderosa sobre nuestro comportamiento cognitivo y emocional causando lo que usualmente llamamos trastornos emocionales. Aunque los hechos activadores (As) a menudo parecen causar directamente a (Cs) no es verdad, porque (Bs) sirve de mediador importante entre (As) y (Cs) es el factor que más directamente causa a (Cs).

Esta terapia sostiene que las personas trasladan sus experiencias a (A) en forma subjetiva y temperamental y que experimentan (A) a la luz de sus creencias y esperanzas; pero también intervienen sus emociones, es decir: deseos, preferencias, gustos y perturbaciones. En consecuencia, los seres humanos nunca experimenta $\mathrm{A} \sin \mathrm{B}$ y $\mathrm{C}$; pero también raramente vivencian $B$ y $C \sin A$. Un procedimiento irracional en nuestra manera de pensar sería el siguiente: 1) lo que hago lo debo hacer perfecto y merecer aprobación, en caso contrario quedo como un fracasado; 2) los demás deben tratarme con consideración y bondad, sino lo hacen la sociedad y el universo deben sentirse culpables y ser castigados por su desconsideración; 3) debo conseguir toda la comodidad que deseo en forma rápida y fácil, nada debe incomodarme.

Asimismo Ellis nos dice que hay cuatro formas de pensamiento autodestructivo que generan problemas psicologicos: 1) Lo terrible: es horroroso que no esté haciendo lo que debo; 2) No puedo soportarlo: no puedo soportar las cosas que me están pasando y que no deben sucederme; 3) Indignidad: soy una persona indigna sino hago las cosas con perfección y me gano la aprobación que debo tener; 4) Falsas generalizaciones: porque he fallado en esta importante tarea, que nunca debería haber sucedido, siempre fallaré y no tendré éxito. Ciertamente las creencias abstractas son a menudo una expresión de la tabla de valores de las personas y juegan un rol importante en sus objetivos de vida. La RET separa las interpretaciones basadas en la realidad de aquellas que se fundamentan en las creencias irracionales que conducen a la perturbación emocional.

La RET plantea 1a siguiente hipótesis de trabajo: cuando (C) consiste en trastonos emocionales tales como: depresión, ansiedad, hostilidad, sentimientos de inferioridad, étc. (B) usualmente (no siempre) está creando (A). Los problemas emocionales, como ya lo hemos mencionado, pueden algunas veces tener su origen en poderosos (A), por ejemplo: desastres sociales y ambientales; o pueden tener su origen en factores orgánicos, disfunciones hormonales, enfermedades, etc. En consecuencia pueden ser de alguna manera independientes de (Bs). Sin embargo, cuando un hecho significativo causa un comportamiento $(\mathrm{C})$, o cuando son factores orgánicos los que motivan (Cs), generalmente el factor (Bs) estuvo presente; por ejemplo si una persona sufre los efectos de una catástrofe o si experimenta disfunciones hormonales y en con- 
secuencia se deprime, consideramos que (As) influyen para producir las creencias irracionales (Bs) tales como: este terremoto nunca debio suceder, ino puedo soportarloi

Estas (Bs) contribuyen a aumentar los sentimientos depresivos expresados en el comportamiento (C). Cuando (s) formado por pensamientos, sentimientos y comportamientos, sigue a (As) y (Bs), (s) no se da en forma pura, sino parcialmente está incluido e interactúa con (A) y (B). Por ejemplo si (A) es un hecho lamentable como ser despedido en el trabajo; (B) es primero una opinión razonable: siempre pensé que serfa una lástima perder este trabajo, pero también puede pensarse en forma irrazonable; soy un fracasado por haber perdido este trabajo, jnunca debí perderlo! Luego (C) primero es un sentimiento normal de frustración y tristeza, pero después se convierte en una opinión irrazonable con sentimientos de ansiedad y depresión.

Además, nos dice Ellis la gente lleva sentimientos, esperanzas y propositos a (A); no se interesan por un trabajo a menos que lo deseen o consideren ventajoso; de esta manera su (A) parcialmente incluye su comportamiento (C); están relacionados entre sí. Al mismo tiempo las creencias de las personas (Bs) incluyen sus (As) y (Cs); de esta manera cuando se dice a sí miasma: deseo conseguir un buen trabajo (B), parcialmente crea la acción (A) por ejemplo sostiene una entrevista y lleva a cabo un comportamiento emocional (C) que puede ser de desencanto y depresión al ser rechazado. Si esta persona no hubiera evaluado el trabajo como bueno, no se hubiese propuesto conseguirlo y luego no expermentarfa frustración al ser rechazado. De tal manera que A, B y C están estrechamente relacionados entre sf y ninguno de ellos existe sin los otros dos. Para esta terapia el ser humano tiene una unidad cognitiva que se combina con sus reacciones emocionales y de conducta; cuando una persona siente y actúa, siempre tiene algunos pensamientos relacionados con sus sentimientos y actitudes. Estos pensamientos lo conducen a expresar otros sentimientos y comportamientos.

Para Ellis cuando las personas se sienten emocionalmente perturbadas (C) están ansiosas, deprimidas y hostiles, observan sus síntomas como algo horroroso y concluyen: ¡no debo estar deprimido! ¡Es horrible que tenga este comportamiento! ¡Soy un tonto al dejarme dominar por este sentimiento! De esta manera desarrollan un segundo síntoma: depresión o ansiedad por su estado ansioso. Síntomas que pueden ser más severos que los primeros. De esta manera bloquean su comprension y limitan su actitud de lucha en sus primeras dificultades. Ciertamente empleamos a menudo en nuestro proceso cognitivo esta manera autodestructiva de pensar. La terapia racional emotiva repara más en los síntomas secundarios o trabaja en forma prioritaria con ellos. El hecho psicológico que las personas tiendan a condenarse a sí mismas cuando experimentan síntomas primarios y posteriormente desarrollen peores sintomas secunda- 
rios, fundamenta la hipótesis de la terapia RET de que la manera como pensamos es sumamente importante en el desencadenamiento de los sentimientos y comportamiento neurótico.

Recordemos que la teoría básica de la RET consiste en afirmar lo siguiente: cuando una persona está seriamente perturbada, está aceptando o inventando dogmáticas creencias irracionales (Bs); uno de los mejores métodos para ayudarle a disminuir o remover sus disturbios emocionales, es enseñarle como debe disputar estos (Bs) en el punto (D); darle valor durante la terapia para que siga con este comportamiento hasta que llegue al punto (E) y adquiera una nueva filosofía que le permita pensar y comportarse en forma más racional e independiente.

La sesión terapéutica se realiza preferentemente mediante la discusión verbal; se insiste en el trabajo, la acción y el esfuerzo personal del paciente. Se utiliza el análisis lógico y la persuasión racional para inducir al paciente a luchar contra sus actitudes neuróticas. Asimismo se le enseña mediante tareas concretas, cómo observar, cuestionar y transformar en reales sus ideas irracionales que motivan sus perturbaciones de personalidad. La técnica tiene presente que estamos condicionados a "agarrarnos firme, enérgica y dramáticamente a nuestras ideas y comportamientos; en consecuencia para experimentar cambios verdaderos es necesario trabajar con mucha firmeza para modificar una conducta perturbada.

Se acostumbra hacer uso de la biblioterapia, dejar tareas escritas con la finalidad de discutir las ideas irracionales; efectuar reforzamientos, sanciones y gratificaciones. El procedimiento durante la aplicación de la técnica es el siguiente:

1) Identificar las creencias irracionales del paciente; 2) Discutir estas creencias; 3) Cambiar los modelos de pensamiento ilógico del cliente y su tendencia a deformar la realidad; 4) Instruirlo sobre las autoafirmaciones racionales apropiadas; y 5) Ensayo y práctica conductual en vivo. El terapeuta no interpreta, sino formula su pregunta en forma clara y explícita; su objetivo es enseñarle al paciente a que aprenda a preguntarse él mismo.

Nos encontramos con un método didáctico, persuasivo y cognitivo. Afirma que transformando el pensamiento del paciente va a cambiar sus emociones. No es una técnica pasiva, se discute y se insiste en el esfuerzo personal y la práctica constante; utiliza el análisis lógico empírico de la ciencia y la persuasión racional para inducir al paciente a trabajar y luchar frente a sus síntomas neuróticos y hábitos destructivos. El terapeuta busca deliberada- 
mente las irracionalidades y se las explica en forma enérgica al paciente a fin de que éste se anime a vencerlas y reemplazarlas por una filosofía de vida más racional. Se confía que esta terapia directiva y persuasiva es eficaz para destruir las causas de las dificultades y conflictos de los clientes. El terapeuta toma una posición no sólo práctica, sino estoica y hedonista.

La actitud del terapeuta es motivar que los pacientes debatan, cuestionan y contra ataquen sus creencias y filosofias autodestructivas consigo mismo y los demás hasta que logren vencerlas. Es necesario, desde el inicio que el trabajo terapéutico sea intenso y de un claro compromiso entre paciente y terapeuta. Es pues una terapia nada contemplativa o condescendiente sino al contrario le va enseñando al paciente el ataque directo de sus ideas destructivas haciéndole ver cómo sus creencias irracionales y su fe en ellas constituye el origen de sus dificultades.

El terapeuta emplea la técnica del comentario egosintónico con la finalidad de levantar el ánimo y la moral del entrevistado y que piense en forma diferente de sí mismo. Insiste en frases que el paciente debe repetir tales como:

¿Por qué darle importancia a lo que piensan de mí?

No es horroroso que me desaprueben

No es terrible que me equivoque. $\mathrm{Si}$ sigo readoctrinándome en mis temores, no cambiaré; en consecuencia lucharé contra mi inercia. Trabajar mis problemas con firmeza y pasión.

La terapia RET interpreta los gestos y actitudes de los pacientes en grado mínimo, su interés se centra en destruir las ideas que se ocultan detrás de las actitudes. Asimismo Ellis afirma que su terapia es más eficaz con pacientes de baja posición economica porque estos esperan que los terapeutas sean activos y directivos.

\section{Conclusión}

Hemos expuesto una síntesis de la terapia racional emotiva fundamentada en las obras principales de Albert Ellis y en la bibliografía pertinente; dejamos para un próximo trabajo la ilustración de la teoría con la casuística respectiva, donde podremos observar cómo los hábitos del pensamiento irracional de los pacientes se hallan muy profundos y tienden a persistir; sólo una práctica permanente logra obtener cambios favorables. 


\section{Referencias}

Adler, (1959). El carácter neurótico. Buenos Aires: Paidós.

Dyer, V. (1980). Técnicas efectivas de asesoramiento psicológico. Barcelona: Grijalbo.

Ellis, (1988). Como vivir con un neurótico. Buenos Aires: Central.

Ellis, (1985). Clinical abplications of rational emotive therapy. New York, Plenun Press.

Ellis. (1981). Manual de terapia racional emotiva. Bilbao: Descless de Brouwer.

Ellis, (1980). Razón y emoción en psicoterapia. Bilbao: Descless de Brouwer Ellis, (1975). A new guide to rational living. New York, Prentice Hall. Ellis, (1974). Psicoterapia racional. En: Millon, Psicopatología y personalidad. México: Interamericana. Pág.: 223-231.

Ellis. (1973). Humanistic psychoterapy: the rational emotive approach. New York: Julian Press.

Frankl, V. (1984). Psicoterapia y Humanismo. México: Fondo de Cultura Económica:

Mahoney, M. J. (1988). Cognición y Psicoterapia. Buenos Aires: Paidós. 